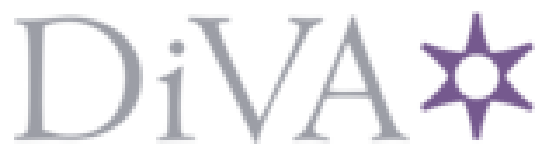

http://www.diva-portal.org

This is the published version of a paper published in .

Citation for the original published paper (version of record):

Sander Tavallaey, S., Saarinen, K. (2009)

Backlash identification in transmission unit

Proceedings of the IEEE International Conference on Control Applications, :

$1325-1331$

Access to the published version may require subscription.

N.B. When citing this work, cite the original published paper.

Permanent link to this version:

http://urn.kb.se/resolve?urn=urn:nbn:se:kth:diva-298583 


\title{
Backlash Identification in Transmission Unit
}

\author{
Shiva Sander-Tavallaey, Kari Saarinen
}

\begin{abstract}
This work presents a methodology identifying the increase of backlash amount in any type of gearboxes for a movable mechanical unit. No external sensor is required. A dedicated test cycle, of at least 4-8 seconds long, is run regularly on the axis under examination. The motion data, i.e. position, velocity and torque references is logged and stored in a file. The file is later on subject for automatic backlash analysis. The sampled data is analyzed and the so called backlash energy, invented in this work, is calculated and stored in a database. The stored data are trended and the criticality of the eventual backlash enlargement is established using a number of statistical methods for the unit under examination. The proposed method is verified in three different experiments on total $19(13+5+1)$ different movable mechanical units and corresponding transmission units with very good results.
\end{abstract}

\section{INTRODUCTION}

$\mathrm{I}_{\mathrm{i} m}^{\mathrm{N}}$ $\mathrm{N}$ today's world of global competition any significant investment in manufacturing requires that the installation shall produce excellent goods quickly and competitively, that non-productive time shall be minimized and that the installation shall communicate easily with external systems for control and management. Good equipment design is essential for processes requiring high reliability. However, no amount of design effort will prevent deterioration over time. Machinery and systems operate under stress in an environment characterized by randomness. Being able to predict with reasonable certainty how much longer a machine can operate safely and properly, we can assure the user of the asset a satisfactory level of reliability.

One of the most important components in different industrial applications is gear transmission. Any faults in the gear transmission can be diverse and depending on the situation can affect the performance of the system considerably. Backlash is a common source of uncertainty found in any geared systems, although some backlash is essential for any gear transmission, less than the appropriate amount results in interference between the teeth whereas excess backlash introduces looseness into the system. In either case, the result is poor performance and possible damage to the system. This effect is most conspicuous when

Manuscript received December 15, 2008

Sh. Sander is with ABB Corporate Research, SE-721 78 Västerås, Sweden. (phone: +46-21-323193; fax: +46-21-323212; e-mail: shiva.sander-tavallaey@se.abb.com).

K. I. Saarinen is with ABB Corporate Research, SE-721 78 Västerås, Sweden. (phone: +46-21-323093; fax: +46-21-323212; e-mail: kari.saarinen (a)se.abb.com). the system is subjected to non-continuous motion with frequent reversal of the direction of rotation.

Backlash measurements are usually tedious, time consuming and thus expensive tasks for service organization. In many cases these costly measurements are performed on several units proactively. Introducing a methodology indicating the criticality of backlash amount ahead of a planned visit will gain time and reduce cost for the service provider as well as the asset-owner.

In the present study we describe the methodology we developed to identify the increase of backlash amount in gearboxes for a movable mechanical unit. No external sensor is required. A dedicated test cycle, of at least 4-8 seconds long, is run on the axis under examination. The motion data, i.e. position, velocity and torque references is logged and stored in a file. The file is later on subject for automatic backlash analysis. The sampled data is then analyzed and the so called backlash energy invented in this work is calculated and stored in a database. The stored data is trended and the criticality of the eventual backlash enlargement is established using a number of statistical methods for the axis under examination. While the backlash enlargement is critical a warning is generated.

In the present paper a brief survey of different approaches in the area of monitoring and fault diagnostics of the gearboxes in recent years is given. Further the signal processing techniques and methods utilized in this work are recounted. Next the investigation phase leading up to the Backlash Energy hypothesis is described. Furthermore the confirmation test of the hypothesis and design of the optimal tests cycles are presented. Finally we end up with discussions and suggestions on future works.

\section{A. State-of-the-art}

In the last decades there have been hundreds of theoretical and practical research papers on developments of different monitoring, diagnostics and prognostics solutions and analysis for mechanical systems in general and rotating machinery in particular, all utilizing various type of measurement techniques and signals. Different types of condition monitoring data has been considered, among which the value type data i.e. data collected at a specific time epoch as single valued variables such as oil analysis data, temperature, pressure, humidity as well as feature values extracted from raw signals via signal processing like r.m.s., peak to peak etc. looks much simpler to process than waveform and/ or image type of data. However, complexity 
lies in the correlation structure when the number of variables is large. Multivariate analysis techniques such as principal component analysis (PCA) and independent component analysis (ICA) are very useful to handle data with complicated correlation structure. Trend analysis techniques such as regression analysis and time series model like AR and ARMA are commonly used techniques for predicting the trend of different value type data, e.g. peak amplitude density, RMS, Kurtosis, MCF of the vibration data, etc. see [1]-[3]. Waveform data i.e. data collected at a specific time epoch as a time series of values includes various types of signals such as motor current, vibration signals and acoustic data etc. There are a large number of analysis approaches utilized analyzing this type of data among which three main groups are: time-domain, frequency- domain and timefrequency analysis.

A popular time-domain analysis approach is time synchronous average (TSA), see [4]-[5]. Further time series modelling such as AR and ARMA aiming to fit the waveform data to a parametric time series model as well as different multivariate statistical techniques have also been utilized in gear fault diagnostics, see [6].

Power spectrum analysis, frequency filters together with different amplitude demodulation or envelope analysis are widely utilized in diagnostic of stationary as well as cyclostationary machine signals, see [7]. Higher order spectrums such as bispectrum analysis, providing more diagnostic information than the power spectrum for nonGaussian signals, have been shown to have wide application in machinery diagnostics for various mechanical systems such as gears, see [8]-[9]. Examples on utilization of parametric modelling approaches in frequency domain i.e. AR and ARMA spectrum in machinery fault diagnostics can be found in [10]-[11].

In order to take care of the non-stationary type of waveform signal generated in machinery fault cases the time-frequency analysis is to be preferred. STFT, Short Time Fourier Transform, see [12]-[13], and Wigner-Ville Distribution (WVD), see [14], have been utilized for gearbox fault detection. Pan et al. 1994 [15] presented an approach in which they did extract features from spectrogram, smoothed WVD and scalogram, detecting and quantifying the backlash in link-joint robot.

Collecting dissimilar partial information on same machine using multi sensors can increase possibilities to a more reliable diagnostic and prognostic analysis on the observed asset. There is a number of data fusion technique utilized in different application. Hall et al. 2004 [16], defined three groups of approaches: data-level, feature-level and decisionlevel. Examples of using data-level as well as feature-level fusion can be found in [17]-[18]. Combining noncommensurate data like oil and vibration for gearbox fault diagnostic and prognostics were shown as a powerful method, although complicated for an on-line condition monitoring, in [19]. One of the major challenges dealing with multi sensor data fusion in mechanical systems is the fact that each and every sensor data has different and multiple sources. Reducing the number of sources, i.e. identifying the "main" source is a challenging task. There are different techniques handling these challenges; blind source separation (BSS) is one of the most popular one, see [20]-[21]. Independent component analysis (ICA) as a special case of BSS is a natural extension of principal component analysis (PCA). Chen et al. [22], applied ICA together with other signal processing tools in helicopter gearbox diagnostic.

The final goal of performing all diagnostics is of course to use the earned knowledge predicting and/ or if possible preventing occurrence of the failures through an adequate decision making. There are several research results hinting ultimate optimized decision strategies. Model-based as well as statistical and Artificial Intelligent approaches are among the most powerful tools for decision making process.

Utilizing the physics specific in model-based approach, leads to explicit mathematical models describing the mechanical as well as dynamical behaviour of the monitored object. Sarkar et al. [23], showed that modelling backlash as a microscopic impact, its presence could be detected and possibly measured using only simple sensors. Several approaches are concentrated to develop models capturing the hysteresis curve characteristic for backlash phenomena, see [24]-[25]. Other approaches in this class are addressed to residual generation methods such as Kalman filter, parameter estimation or system identification and parity relations, see [26]-[27].

Among statistical approaches the conventional SPC-statistical process control-- has been used widely in different applications of fault detection and diagnostics; see [3] and [28]. Clustering analysis is another type of statistical methods aiming to identify and isolate different faults. The idea here is to maximize the difference between different groups of faults minimizing the deviation within a certain group. There are several discriminant functions conceivable among which the Euclidean and Bayesian distances are the most common one, see [29]. Correlation coefficients relating different property vectors are also exposed as a similarity measure in machinery fault diagnosis; see [30]. Furthermore the decision making and fault diagnostic can be formulated as a hypothesis test. Using different statistical models and distributions can these hypotheses be expressed and analyzed. M. Nyberg [31], showed how to handle complex systems with several faults presented using a general framework, based on structured hypothesis tests.

Artificial Neural Network (ANN) and Expert System (ES) are the most popular approaches in the AI category of decision making applied to fault diagnostics of rotary machines. A review of recent developments in applications of AI techniques for induction machine stator fault diagnostics was given by Siddique et al [32]. 


\section{MATHEMATICAL METHODS}

Mechanical movable units and gearboxes under test create lots of data. In previous chapter we presented a review of different types of algorithms develop for gearbox analyses and diagnostics. For methods that should be put on practise the complexity is not the end in itself; on the contrary the simpler the better. Due to the restrictions on number of pages only the methods that turned out to be most useful are shortly presented in this chapter.

\section{A. Spectral estimator for finite data sequence}

Due to cyclic nature of the signals produced by cyclic dynamic forces in gearbox it is advantageous to use frequency domain analysis techniques, in which the fundamental idea is to find the relation between spectral component frequency and the frequencies of the dynamic forces producing the faults.

Power spectral density (PSD) of a wide sense stationary (WSS) random process $x[n]$ is defined as discrete-time Fourier transform of the autocorrelation sequence [33].

The alternative formal definition of the PSD, based on ergodicity, has the discrete time form called periodogram spectral estimator:

$$
P(f)=\frac{1}{N}\left|\sum_{n=0}^{N-1} x[n] \exp (-j 2 \pi f n)\right|^{2}
$$

for a finite data sequence $x[n]$ of $N$ samples.

The most common way to improve statistical properties of the periodogram, is to calculate an averaged periodogram of windowed and overlapped data segments (Welch periodogram [34]).

\section{B. Fourier analysis of pulse trains}

Local defects as cracked tooth in gear produce a series of impacts (or shock pulses) which repeat periodically at the rate relative to the shaft speed.

The following mathematical analysis helps to understand the formation of the spectrum of any pulse train. Assume $M$ coherent pulses in noiseless environment [35]

$$
B(t)=M^{-1 / 2} \sum_{k=0}^{M-1} b\left(t-k T_{r}\right),
$$

where $T_{r}$ is time between two pulses, and $b(t)$ a single pulse. The Fourier transform of $B(t)$ is

$$
\begin{aligned}
B(f) & =M^{-1 / 2} \int_{-\infty}^{+\infty}\left[\sum_{k=0}^{M-1} b\left(t-k T_{r}\right)\right] \exp (-j 2 \pi f t) d t \\
& =M^{-1 / 2} P(f) C(f) \exp \left[-j \pi(M-1) T_{r} f\right]
\end{aligned}
$$

where

$$
P(f)=\int_{-\infty}^{+\infty} b(t) \exp (-j 2 \pi f t) d t
$$

is the Fourier transform of the single pulse and

$$
C(f)=\frac{\sin \left(M \pi T_{r} f\right)}{M \sin \left(\pi T_{r} f\right)},
$$

is so called comb function (Fig. 1). The comb function consists of peaks of height equal to one and that are separated in frequency by $1 / T_{r}$, whose widths are the order of $1 /\left(M T_{r}\right)$.

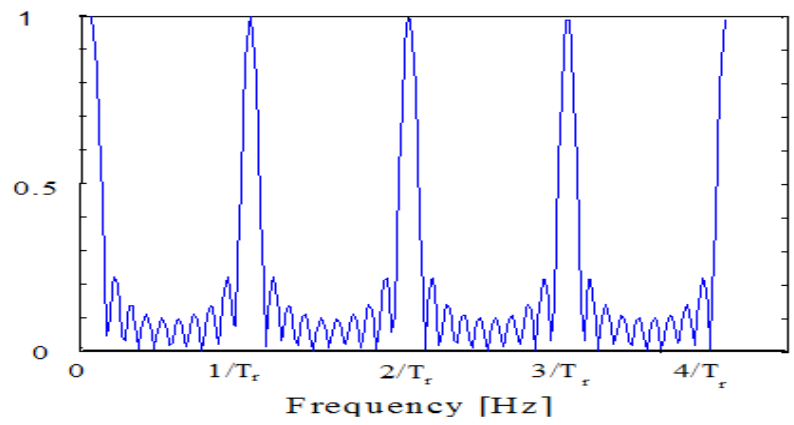

Fig. 1 The comb function.

\section{Binary decision}

An automatic fault detection algorithm should analyze the measured signal by calculating characteristic values and also make a decision if there are any indication of fault in the measured data or not. As a random phenomenon, the signal must be analyzed in the framework of the theory of probability.

Viewed in the most elementary way, the task of the backlash detection algorithm is to choose between two hypotheses:

$$
\begin{aligned}
& H_{0}: x(t)=n(t) \quad \text { (only random noise) } \\
& H_{1}: x(t)=s(t)+n(t) \quad \text { (presence of backlash and noise) }
\end{aligned}
$$

The algorithm is said to make a binary decision.

Classical statistical process control (SPC) tools [36] offer an easy-to-understand and well defined way to implement binary decision theory in practice. For example so called $\bar{x}$ and $\mathrm{R}$ charts (Shewhart charts) can be used to decide if the system is in normal operation conditions or not. If the measured value is between upper and lower control limits (UCL and LCL) the system is in normal conditions. However the normal goal to avoid large number of false alarms causes the major disadvantage of any Shewhart chart. If the shift in the measured values is in the range of \pm 1.5 times standard deviation $(\sigma)$, then the Shewhart charts will not capture the changes in the process. When detection of these small ascending changes are of interest, then the cumulative-sum (CUSUM) or exponentially-weightedmoving-average (EWMA) are more effective alternatives.

Because EWMA can be viewed as weighted average of all past and current observations, it is very insensitive to normality assumption. It is therefore an ideal chart to use with individual observations.

\section{EXPERIMENTAL RESULTS}

Three types of experiments were performed. The first test group consisted of 13 mechanical movable units of type 1 with various health conditions. The second test consisted of 1 unit of type 2 at our research center laboratory with and without synthetically introduced backlash. Finally 
accelerated lifetime measurements were performed on five gearboxes of type 1 .

\section{A. Primary investigation}

Thirteen mechanical movable units with different health condition were tested. The tested units were 7 years old but with different operation time.

The test cycles run at each and every individual axis in the first approach, were identical with slightly different size of position angels and at three different velocities. Motion data was logged with $1.984 \mathrm{kHz}$ sampling frequency. Fig. 2 depicts the input sequence consisted of six pulses logged in 35 seconds for axis 1 with $150 \mathrm{rads} / \mathrm{s}$ speed.

Fig. 2. The logged motion data: input sequence, speed and torque.

We applied different signal processing methods on the test case results and observed differences in spectra of the torque signal. Figures 3 and 4 show Welch spectra of torque signals. One can see that there are no big differences between these spectra with exception around second harmonic of rotation speed $(47.5 \mathrm{~Hz})$.

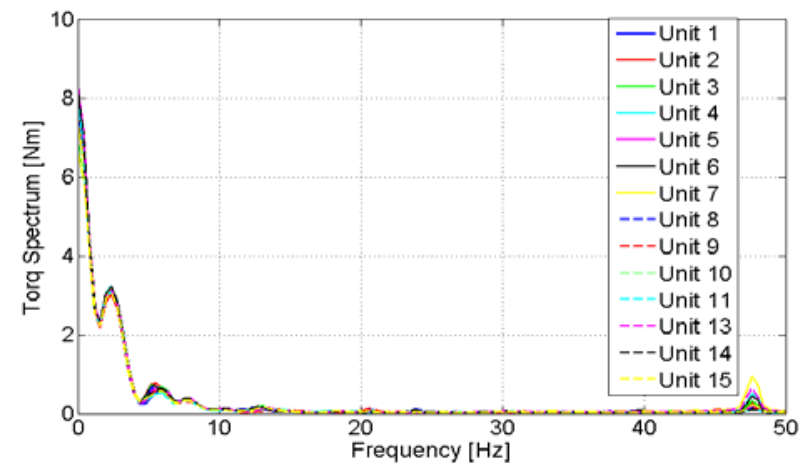

Fig. 3. The Welch spectra the torque signals.

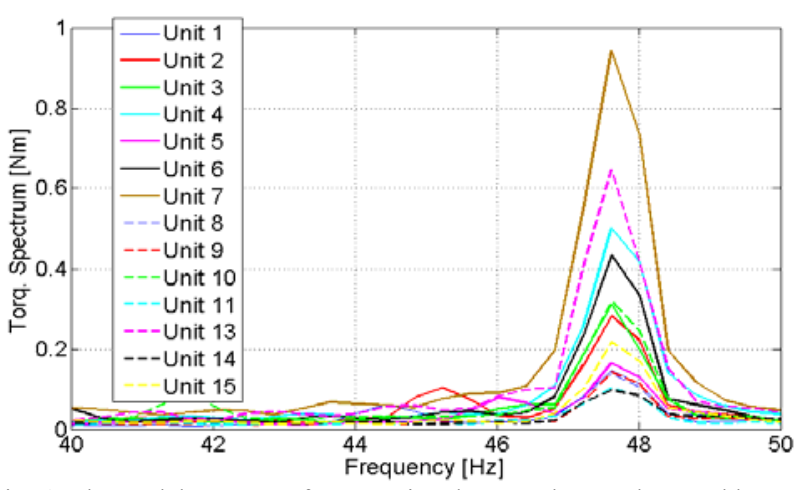

Fig. 4. The Welch spectra of torque signal zoomed around second harmonic of the rotation speed.

The observed amplitude difference around second harmonic among the tested units could clearly be addressed to the measured backlash on corresponding axes. In order to examine whether this deviation can be used as a measure for backlash identification and / or detection corresponding analysis was performed on our test unit. For this purpose backlash was synthetically introduced in the gearbox on our laboratory through increasing the tolerance between driving shaft of the motor and the driven pinion in the gearbox. The backlash was measured using a conventional measurement instrument, both before and after. The result of these measurements showed an increase of play by approximately $12 \%$.

A spectrum analysis of the torque signal before and after introducing backlash indicated an increase of energy amount around second harmonic of the rotation speed (Fig. 5). By integrating the spectrum in the frequency band around second harmonic of the rotation speed we could obtain $15 \%$ increase in energy amount. We have named this characteristic of energy as backlash index (BI) and the corresponding method BI-method.

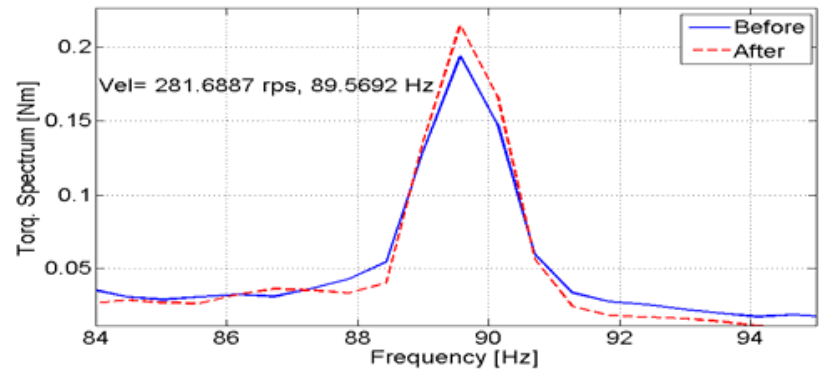

Fig. 5. Torque spectrum, zoomed around second harmonic.

\section{B. Problem related to regular test cycle}

In all the above mentioned anayses we used Welch method to calculate spectra. When studying the low frequency area and the area around the second harmonic of the rotation speed of the torque spectra without any window function and with maximum frequency resolution (37 500 frequency points) we observed evenly spaced peaks in spectra. Figure 6 depicts the low frequency part of the line spectrum of the torque signal of the 13 gearboxes for units 
under consideration. One can clearly see the peaks multiple of $0.366 \mathrm{~Hz}$. The zoomed spectra of the same signals around second harmonic of the rotation speed are shown in Figure 7. One can notice that the even harmonics of $0.1829 \mathrm{~Hz}$ are quite distinguishable in this frequency range.

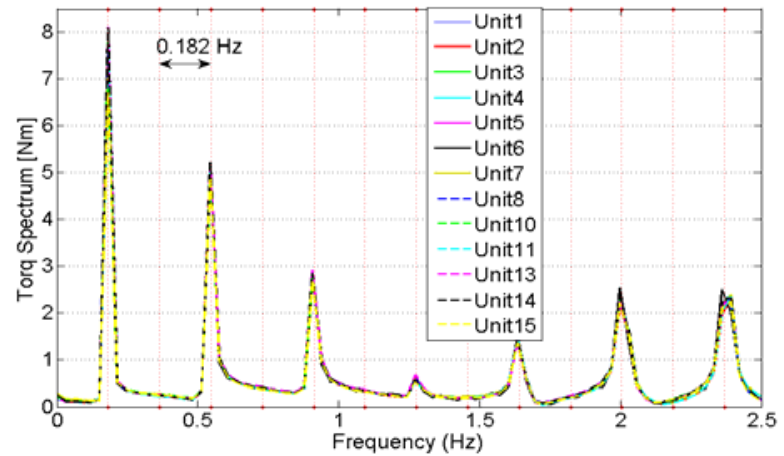

Fig. 6. Low frequency part of the spectra of torque signals. Data logged on gearboxes of type 1 units. The vertical dashed lines show the peaks of the comb function.

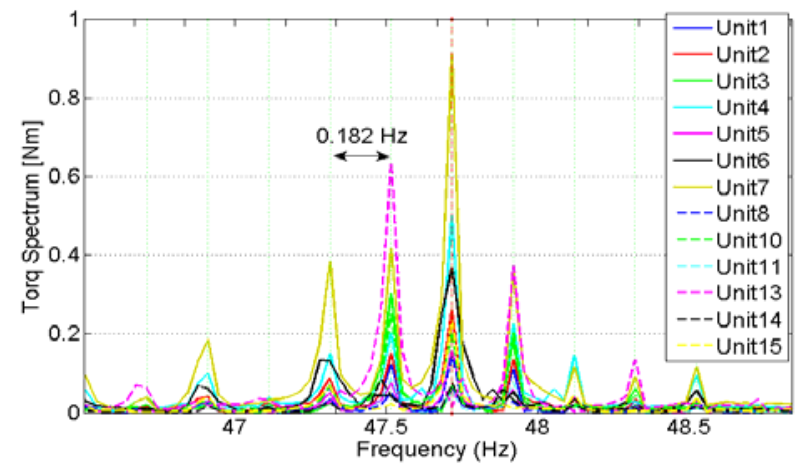

Fig. 7. Zoomed spectra of torque signals around the second harmonics of the rotation speed. Data logged on gearboxes of type 1units. The vertical dashed lines show the peaks of the comb function

We discovered that these spikes in spectra are result of non-optimal input signal. Fourier transform of this sequence of coherent pulses is product of the comb function and the corresponding Fourier transform of a single pulse (see Eq. 3). Peaks of the comb function are separated in frequency by $1 / T_{r}$, where $T_{r}$ is the length of the pulse, here $5.48 \mathrm{~s}$. Thus, the frequencies of the peaks of the comb function are harmonics of $0.182 \mathrm{~Hz}$. The Fourier series of an input pulse is

$600 / \pi\left[\sin \left(2 \pi / T_{r}\right)+1 / 3 \sin \left(6 \pi / T_{r}\right)+1 / 5 \sin \left(10 \pi / T_{r}\right)+\cdots\right]$.

Note that amplitudes of the even terms are zeros (see also Fig. 6). Furthermore the spectrum of an output signal of a linear system is product of frequency response function of the system and spectrum of an input signal. This means that if the input signal does not contain certain frequency, the frequency will not show up in the output signal either.

One explanation why there are also spikes at the frequencies of the even terms of the Fourier series in higher frequencies (Fig. 7) is that the input sequence is not perfect.
This means that the input signal with regular pulses should preferably be avoided in order to minimize the unwanted disturbances and thus loss of the intresting information.

\section{Tests with new test cycle}

New dedicated cycles with pseudo randomly generated pulses were composed. Backlash measurements were performed on all units and corresponding gearboxes using a conventional backlash measurement tool. Two movable units with highest measured amount of backlash on axis 1 and 2 respectively were utilized for the new test cycles followed by frequency analysis. Once again we observed higher amplitude level of the spectrum around the second harmonic of rotation frequency, for unit 1 axis 1 and unit 2 axis 2 . A comparison between the calculated results using BI method and the corresponding measured backlash is shown in Figure 8.

BI method is consistent with the conventional measurement method. BI increases with increasing measured value. In addition, the relative increase is larger for axis 2 for both of the methods. However, BI method is more sensitive since the ratio of the BI calculated for more and less backlash case is at least double than the corresponding ratio of measured values.

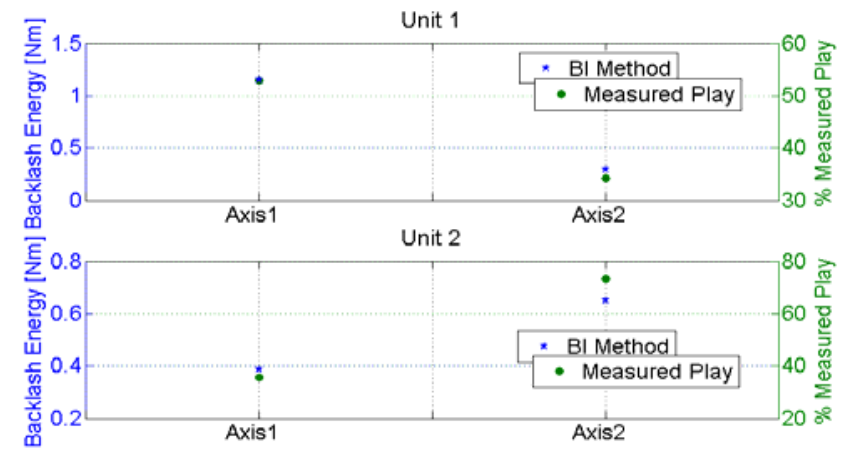

Fig. 8. A comparison between BI method and conventional backlash measurement tool applied on unit 1 and unit 2 and the corresponding axis 1 and axis 2 of these units.

\section{Accelerated lifetime tests}

The main objective of these measurements from backlash identification point of view was to examine the capability of BI- method as an on-line CM tool in the field. Accelerated lifetime measurements were performed on 6 movable mechanical units of type 1 . The considered gearboxes differed greatly in health condition. The test cycles consisted of randomly generated movements according to the experiments earned from former measurements. The length of the signal, i.e. the time window was shortened.

BI was measured once in a day on the "warm" and once in a week on the "cold" status of the units using improved test cycle. The traditional backlash measurements this time using another in-house developed tool were carried out twice a week for cold and warm status respectively.

The calculated backlash energy indices were trended applying the traditional SPC, CUSUM and EWMA. Fig. 9 
shows the result of a traditional SPC analysis in a time window of approximately 4 months.

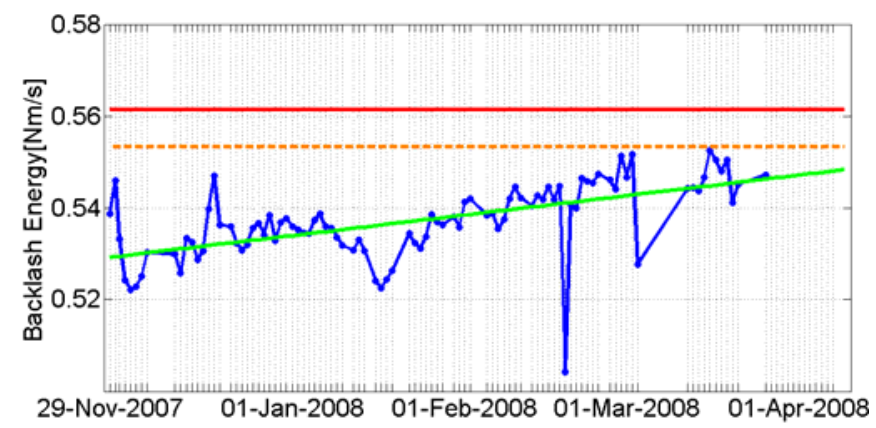

Fig. 9. The calculated backlash energy indices, the blue markers, and the corresponding lower and upper warning level, dashed and solid red lines, for the current population estimated using the traditional SPC.

The correlation coefficient between the estimated backlash energy BI and loss-motion measurements using the in-house measurement tool was quite satisfactory, 0.88 . This is in spite of the fact that the repeatability of the in-house measurement tool was not as accurate as the conventional backlash measurement tool used in previous case. The standard deviation of the measurements with the in-house tool was measured to $15 \%$.

Among the other aspects to be considered is the influence of the temperature on the performance of the suggested method. Comparison of BI measured from "cold" and "warm" transmission units showed that the method is almost non-sensitive to the temperature variation.

\section{CONCLUSION}

The original aim of this work was to investigate the possibility of developing a method for monitoring the enlargement of the backlash amount in compact type of gearboxes. The restricting requirements such as: avoidance of using external sensors, the amount of data to be transferred should be minimized in size and finally the fact that the interference on the normal operation of the observed unit in the field should be minimized in time and space, made the use of conventional methods, even if such methods exist, less attractive and the task more challenging.

The present method was developed in a short time window combining the experiments with available and well known methodologies and experiences in the area of condition monitoring and diagnostics of rotating machinery.

It is well-known that the second harmonic of the rotation speed is dominated in the vibration signal spectrum of rotating machinery when the misalignment is presented in the system. In other words the misalignment results to a localized energy increase in the second harmonic frequency band. Assuming that the increase of backlash in compact type of gearboxes introduces misalignment in between main crank shaft and gearbox, one can expect the same behaviour in the vibration signal on the gearbox and in best case even in the motor torque signal.
This energy here called as "backlash energy $(\mathrm{Nm} / \mathrm{s})$ "is defined as energy generated around the second harmonic of the rotation speed traceable in spectrum of the torque signal. Due to the fact that the velocity is not constant in our application we need to integrate energy around the estimated average frequency of interest.

Overall, the results indicate that, at a relatively low computational cost, the developed method can capture the variation of the backlash phenomena in the compact gearboxes. The method was qualitatively compared with two different backlash measurement tools with good results. A limited temperature sensitivity analysis indicated the robustness of the method.

The choice of the input sequence was shown to play a critical role for the analysis. In order to capture the phenomena optimally a dedicated test cycle should be run at the movable mechanical unit.

Furthermore the decision making part of backlash identification procedure in context of condition monitoring approach is performed applying the traditional SPC, CUSUM and EWMA.

This methodology is not to be exchanged as a quantitative tool in traditional type of backlash measurements. The method should be used as a complementary to the one. Though backlash measurements are usually a tedious, time consuming and thus expensive tasks for service organization and in many cases are performed on several units proactively, the suggested methodology in this report indicating the criticality of backlash amount ahead of a planned visit will gain time and reduce cost for the service organization as well as the customer.

A number of interesting further studies suggest themselves. Firstly, a more comprehensive parameter tuning in decision making procedure would be appropriate. Another useful extension of this work would be to investigate a rigorous optimization scheme of the sampling frequency as well as length of the measurement and the input sequence.

\section{REFERENCES}

[1] G. O. Allgood, B. R. Upadhyaya. A model-based high-frequency matched filter arcing diagnostic system based on principal component analysis (PCA) clustering. in: Applications and Science of Computational Intelligence III. 4055. Bellingham, 2000. pp. 430-440.

[2] B. K. Sinha. Trend prediction from steam turbine responses of vibration and eccentricity. Proceedings of the Institution of Mechanical Engineers Part A-Journal of Power and Energy. 216 (2002) 97-104.

[3] M. Tunklev. 9ADB000039-023-The Wear and Q Calculations with Residual Lifetime Estimation. SECRC/ AT- 2006.

[4] G. Dalpiaz, A. Rivola, R. Rubini. Effectiveness and sensitivity of vibration processing techniques for local fault detection in gears. Mechanical Systems and Signal Processing. 14 (2000) 387-412.

[5] A. J. Miller. A New Wavelet Basis For The Decomposition Of Gear Motion Error Signals And Its Application To Gearbox Diagnostics, M.Sc. Thesis. Graduate Program in Acoustics. The Pennsylvania State University, State College, PA. 1999.

[6] N. Baydar, Q. Chen, A. Ball, U. Kruger. Detection of incipient tooth defect in helical gears using multivariate statistics. Mechanical Systems and Signal Processing. 15 (2001) 303-321. 
[7] R. B. Randall, J. Antoni, S. Chobsaard. The relationship between spectral correlation and envelope analysis in the diagnostics of bearing faults and other cyclostationary machine signals. Mechanical Systems and Signal Processing. 15 (2001) 945-962.

[8] L. Xiong, T. Shi, S. Yang, R. B. K. N. Rao. A novel application of wavelet- based bispectrum analysis to diagnose faults in gears. International Journal of COMADEM. 5 (2002) 31-38.

[9] W. Li, G. Zhang, T. Shi, S. Yang. Gear crack early diagnosis using bispectrum diagonal slice. Chinese Journal of Mechanical Engineering (English Edition). 16 (2003) 193-196.

[10] J. P. Dron, L. Rasolofondraibe, C. Couet, A. Pavan. Fault detection and monitoring of a ball bearing bench test and a production machine via autoregressive spectrum analysis. Journal of Sound and Vibration. 218 (1998) 501-525.

[11] M. J. E. Salami, A. Gani, T. Pervez. Machine condition monitoring and fault diagnosis using spectral analysis techniques. in: Proceedings of the First International Conference on Mechatronic (ICOM '01). Vol. 2. Kuala Lumpur, Malaysia, 2001. pp. 690-700.

[12] W. J. Wang, P. D. McFadden. Early detection of gear failure by vibration analysis I. Calculation of the time-frequency distribution. Mechanical Systems and Signal Processing. 7 (1993) 193-203.

[13] F. A. Andrade, I. Esat, M. N. M. Badi. Gearbox fault detection using statistical methods, time-frequency methods (STFT and Wigner-Ville distribution) and harmonic wavelet - A comparative study. in: Proceedings of COMADEM '99. Chipping Norton, 1999. pp. 77-85.

[14] M.-C. Pan, H. Van Brussel, P. Sas, B. Verbeure. Fault diagnosis of joint backlash. Journal of Vibration and Acoustics. Transactions of the ASME. 120 (1998) 13-24.

[15] M.-C. Pan, H. Van Brussel, P. Sas, B. Verbeure. Dtection and quantification of the Link-Joint Backlash of a Robot-Time-Frequency Signal Representation and Feature Extraction- Proceedings of the International conference on Noise and Vibration Engineering ISMA 19, Sep. 1994. pp. 407-421.

[16] D. L. Hall, S. A. H. McMullen. Mathematical techniques in multisensor data fusion. Artech House, Boston. 2004.

[17] T. Heger, M. Pandit. Optical wear assessment system for grinding tools. Journal of Electronic Imaging. 13 (2004) 450-461.

[18] Q. Liu, H.-P. Wang. A case study on multisensor data fusion for imbalance diagnosis of rotating machinery. (AI EDAM) Artificial Intelligence for Engineering Design, Analysis and Manufacturing. 15 (2001) 203-210

[19] C. S. Byington, T. A. Merdes, J. D. Kozlowski. Fusion techniques for vibration and oil debris/quality in gearbox failure testing. in: Proceedings of Condition Monitoring '99, Chipping Norton. 1999. pp. 113-128.

[20] Xianhua Liu and R.B. Randall. Blind source separation of internal combustion engine piston slap from other measured vibration signals. Mechanical Systems and Signal Processing. Volume 19, Issue 6. November 2005. Pages 1196-1208.

[21] C. Serviere, P. Fabry. Blind source separation of noisy harmonic signals for rotating machine diagnosis. Journal of Sound and Vibration. 272 (2004) 317-339.

[22] Z. S. Chen, Y. M. Yang, G. J. Shen, X. S. Wen. Early diagnosis of helicopter gearboxes based on independent component analysis. in: Proceedings of ISTM/2003: 5th International Symposium on Test and Measurement. Vol. 1-6. Beijing, 2003. pp. 3383-3386.

[23] N. Sarkara, R. E. Ellisb and T. N. Moore. Backlash detection in geared mechanisms: modeling, simulation, and experimentation. Journal of Mechanical Systems and Signal Processing, Volume 11, Issue 3, 3 May 1997. pp. 391-408.

[24] P.Ge, M. Jouaneh. Modeling of the Backlash Hysteresis Nonlinearity. Journal of Mechanical Design, ASME, Vol.120, Sep. 1998. pp. 408413.

[25] S. Dubrowsky, F. Freudenstein. Dynamic analysis of mechanical sytems with clearances, Part 1: Formation of Dynamic model. Journal of Engineering for industry, ASME, Feb. 1971. pp. 305- 309.

[26] W. Y. Wang. Towards dynamic model-based prognostics for transmission gears. in: Component and Systems Diagnostics, Prognostics, and Health Management II, 4733, Bellingham, 2002, pp. 157-167.
[27] André C. Bittencourt. Friction change detection in industrial robot arms. 9ADB001225-041 KTH Msc Thesis (Andre Bittencourt).pdf. SECRC/ AT, 2007

[28] M. L. Fugate, H. Sohn, C. R. Farrar. Vibration-based damage detection using statistical process control. Mechanical Systems and Signal Processing, 15 (2001) 707-721.

[29] W. J. Staszewski, K. Worden, G. R. Tomlinson. Time-frequency analysis in gearbox fault detection using the Wigner-Ville distribution and pattern recognition. Mechanical Systems and Signal Processing, 11 (1997) pp. 673-692.

[30] X. Lou, K. A. Loparo. Bearing fault diagnosis based on wavelet transform and fuzzy inference. Mechanical Systems and Signal Processing, 18 (2004) 1077-1095.

[31] M. Nyberg. A general framework for fault diagnosis based on statistical hypothesis testing. in: Twelfth International Workshop on Principles of Diagnosis (DX 2001). Via Lattea, Italian Alps, 2001. pp. 135-142.

[32] A. Siddique, G. S. Yadava, B. Singh. Applications of artificial intelligence techniques for induction machine stator fault diagnostics: Review. in: Proceedings of the IEEE International Symposium on Diagnostics for Electric Machines, Power Electronics and Drives. New York, 2003. pp. 29-34.

[33] S. M. Kay, Modern Spectral Estimation: Theory and Application. Prentice-Hall, Englewood Cliffs, New Jersey, 1988, pp. 543.

[34] L. S. Marple, Jr, Digital Spectral Analysis with Applications. PrenticeHall. Englewood Cliffs, New Jersey, 1987, pp. 492.

[35] C.W. Helstrom, Elements of Signal Detection and Estimation. Prentice-Hall, Englewood Cliffs, New Jersey, 1995, pp. 586.

[36] D.C. Montgomery, Introduction to Statistical Process Control. John Whiley\&Sons, New York, 1990, pp. 674 